\title{
BMJ Open Feasibility of individualised severe traumatic brain injury management using an automated assessment of optimal cerebral perfusion pressure: the COGiTATE phase II study protocol
}

To cite: Beqiri E, Smielewski P, Robba C, et al. Feasibility of individualised severe traumatic brain injury management using an automated assessment of optimal cerebral perfusion pressure: the COGiTATE phase II study protocol. BMJ Open 2019;9:e030727. doi:10.1136/ bmjopen-2019-030727

- Prepublication history and additional material for this paper are available online. To view these files, please visit the journal online (http://dx.doi. org/10.1136/bmjopen-2019030727).

Received 30 March 2019 Revised 23 July 2019 Accepted 21 August 2019

Check for updates

(C) Author(s) (or their employer(s)) 2019. Re-use permitted under CC BY-NC. No commercial re-use. See rights and permissions. Published by BMJ.

For numbered affiliations see end of article.

Correspondence to

Dr Erta Beqiri;

erta.beqiri@gmail.com

\section{ABSTRACT}

Introduction Individualising therapy is an important challenge for intensive care of patients with severe traumatic brain injury (TBI). Targeting a cerebral perfusion pressure (CPP) tailored to optimise cerebrovascular autoregulation has been suggested as an attractive strategy on the basis of a large body of retrospective observational data. The objective of this study is to prospectively assess the feasibility and safety of such a strategy compared with fixed thresholds which is the current standard of care from international consensus guidelines.

Methods and analysis CPPOpt Guided Therapy: Assessment of Target Effectiveness (COGiTATE) is a prospective, multicentre, non-blinded randomised, controlled trial coordinated from Maastricht University Medical Center, Maastricht (The Netherlands). The other original participating centres are Cambridge University NHS Foundation Trust, Cambridge (UK), and University Hospitals Leuven, Leuven (Belgium). Adult severe TBI patients requiring intracranial pressure monitoring are randomised within the first 24 hours of admission in neurocritical care unit. For the control arm, the CPP target is the Brain Trauma Foundation guidelines target $(60-70 \mathrm{~mm} \mathrm{Hg})$; for the intervention group an automated CPP target is provided as the CPP at which the patient's cerebrovascular reactivity is best preserved (CPPopt). For a maximum of 5 days, attending clinicians review the CPP target 4-hourly. The main hypothesis of COGiTATE are: (1) in the intervention group the percentage of the monitored time with measured CPP within a range of $5 \mathrm{~mm} \mathrm{Hg}$ above or below CPPopt will reach $36 \%$; (2) the difference in between groups in daily therapy intensity level score will be lower or equal to 3 . Ethics and dissemination Ethical approval has been obtained for each participating centre. The results will be presented at international scientific conferences and in peer-reviewed journals.

Trial registration number NCT02982122

\section{INTRODUCTION}

The main aim of the intensive care management of severe traumatic brain injury (TBI)
Strengths and limitations of this study

- First randomised controlled trial prospectively evaluating the feasibility and effectiveness of targeting a cerebral autoregulation-guided cerebral perfusion pressure (CPP) management in severe traumatic brain injury patients.

- For a maximum of 5 days, every 4 hours the attending clinicians are asked to review the next CPP target, through a provided custom made bedside software. Clinicians are allowed to overrule the suggested targets in both study arms.

- The adjusted CPPopt calculation algorithm and the study custom made software module are presented.

- The study was powered to accomplish a relative increase of $20 \%$ of monitored time with CPP close to individual CPPopt target.

- The study is not designed to assess differences in clinical outcome.

patients is to prevent secondary injury. Central to this is the control of intracranial pressure (ICP) and maintaining an adequate cerebral perfusion pressure (CPP). ${ }^{1}$ Various international guidelines have recommended a variety of CPP targets over the years with the current iteration of Brain Trauma Foundation (BTF) 2016 guidelines recommending a CPP tightly controlled between 60 and $70 \mathrm{~mm}$ $\mathrm{Hg}^{2}$

However, it is unlikely that such a fixed therapeutic CPP target will be optimal for all individual TBI patients. Indeed, a failure to individualise therapies has been suggested as a reason for the difficulty to translate plausible therapies into outcome benefit. ${ }^{1}$ With the demographics of TBI changing to an older patient group with more comorbidity, 
the patient group is likely to become more heterogeneous. ${ }^{3}$ Furthermore, TBI pathobiology evolves ${ }^{4}$ and so, even within the same individual, optimal targets may vary over time. Potentially, individualising therapies and tailoring medical treatment to dynamic pathophysiology could offer a precision medicine approach with advantages over the current 'one size fits all' strategy.

In health, cerebral autoregulation (CA) maintains a constant and adequate cerebral blood flow, thereby protecting the brain from both ischaemia and hyperperfusion in the face of inevitable changes in CPP. CA can frequently be impaired after TBI and this is related to poor outcome..$^{5-7}$ Czosnyka et al first demonstrated that the moving correlation coefficient (pressure reactivity index, PRx) between slow changes in arterial blood pressure (ABP) and ICP offers a surrogate method for the continuous bedside estimation of global CA. ${ }^{8}$

This concept has gained interest in the last decade with the observation that PRx and CPP often exhibit a U-shaped relationship over time with a minimum PRx occurring at a CPP for which cerebrovascular pressure reactivity is best preserved (or least impaired). ${ }^{9}$ Such observations suggest that targeting a CPP such that global CA is best maintained ('optimal' CPP-CPPopt) is a potentially attractive strategy for individualising care.

In recent years, there has been a great deal of work in trying to translate the concept CPPopt into an automated clinical application at the bedside. ${ }^{9-12}$ Such technological improvements have been accompanied by a growing body of retrospective evidence (mostly single centre) of a robust association between poor outcome and deviation from calculated CPPopt in severe TBI patients. ${ }^{9} 10$ Prospective evaluation has, however, been lacking to date. Two prospective pilot studies evaluating autoregulation/ CPPopt tailored therapy in different settings demonstrated an improvement in patient outcome. ${ }^{13}{ }^{14}$ However, neither was a randomised study with a published intervention protocol. Prospective evaluation is therefore urgently needed.

A phase II study is needed to resolve a number of outstanding issues before a properly powered and designed phase III trial can be conducted. In particular, it is not known whether it is feasible to track a dynamically changing physiological target in practice. Furthermore, retrospective studies have demonstrated that CPPopt tends to be higher than the $60-70 \mathrm{~mm} \mathrm{Hg}$ fixed target from international guidelines. ${ }^{10}{ }^{12}$ The physiological effects of this are difficult to assess from retrospective data. Targeting higher CPP values may require a higher therapeutic intensity (eg, greater use of vasopressors and fluids) and lead to extracranial complications (eg, pulmonary, myocardial or renal injury). In addition, the very intervention of targeting CPPopt may itself affect the ICP behaviour, in terms both of mean values and presence of transient hypertensive episodes, and in general on brain compensatory reserve and compliance.

In summary, a prospective evaluation of the feasibility, safety and the physiological implications of CPPopt-guided management is now timely to inform the design of any future phase III study in severe TBI patients with ICP monitoring. In this paper we describe the protocol for such a phase II study: 'CPPOpt Guided Therapy: Assessment of Target Effectiveness (COGiTATE)'.

\section{METHODS AND ANALYSIS \\ Design}

COGiTATE is a prospective, multicentre, non-blinded, randomised, controlled trial in patients with severe TBI. Three tertiary centres are involved at the start of the study: Maastricht University Medical Center, Maastricht (The Netherlands), Cambridge University Hospitals NHS Foundation Trust, Cambridge (UK), and University Hospitals Leuven, Leuven (Belgium).

The patients are recruited in the first 24 hours of admission in the intensive care unit (ICU) and randomised into two treatment arms: the control group (CPP target given by the BTF guidelines- $60-70 \mathrm{~mm} \mathrm{Hg}$ ) and the intervention group (CPP target given by the autoregulation status-CPPopt). The duration of the study is 5 days. Other interventions (such as measures to control ICP) are unaltered from local protocols.

The first patient was enrolled on 16 February 2018.

\section{Objectives}

The main objective of the study is to demonstrate that targeting CPP at CPPopt is feasible in TBI patients. The secondary objective is to demonstrate that targeting CPP at CPPopt is safe in TBI patients. The primary endpoints are (1) feasibility: the percentage of monitored time with measured $\mathrm{CPP}$ within a range of $5 \mathrm{~mm} \mathrm{Hg}$ above or below CPPopt; (2) safety: daily therapy intensity level (TIL) score. The secondary endpoints are safety and physiology variables listed in box 1 .

The main hypothesis of COGiTATE are: (1) in the intervention group the percentage of the monitored time with measured $\mathrm{CPP}$ within a range of $5 \mathrm{~mm} \mathrm{Hg}$ above or below CPPopt will reach $36 \%$; (2) the difference in between groups in daily TIL score will be lower or equal to 3 .

\section{Methods and measurements}

Data collection and online processing

In this study, ICP and ABP will be monitored as per normal clinical practice in the units: ICP will be recorded by a parenchymal ICP probe; ABP will be monitored by invasive arterial cannulation in the radial artery and zeroed at the level of the foramen of Monroe. The data will be collected at a frequency $>100 \mathrm{~Hz}$ (waveform resolution), and processed by means of ICM+ ${ }^{\circledR}$ software (http:// icmplus.neurosurg.cam.ac.uk) at the bedside.

\section{COGiTATE CPPopt algorithm}

PRx is calculated as a moving correlation coefficient between $10 \mathrm{~s}$ averages of ICP and ABP waveforms in a window of $5 \mathrm{~min}$. CPPopt is calculated using a multiwindow approach inspired by Depreitere et $a l,{ }^{15}$ subsequently 


\section{Box 1 Prespecified secondary outcomes}

\section{Safety variables}

- ICP/CPP variability (representing adequacy/consistency of ICP control).

- Frequency and average duration of ICP spikes $>20 \mathrm{~mm} \mathrm{Hg}$ (associated with a dose-dependent harm).

- Frequency and average duration of CPP outside preset safety ranges (50-100 mm Hg).

- Mean daily RAP (correlation between ICP amplitude and ICP) index (as measure of brain compensatory reserve).

- Analysis of routine CT head scans for evidence of differences in contusion, cerebral oedema and haemorrhage.

- Mean daily vasopressor dose; necessity and dose of inotropic support.

- Incidence of troponin rise and ECG changes stratified by day (as measure of cardiac complications).

- Incidence of serum creatinine rise (as measure of renal complications).

- Mean daily fluid balance.

- Mean daily oxygen arterial pressure/oxygen inspired fraction $\left(\mathrm{PaO}_{2} / \mathrm{FiO}_{2}\right)$ ratio + oxygenation index (as measure of pulmonary complications).

- Intensive care survival, and Glasgow Coma Score at ICU discharge.

\section{Physiology variables \\ - Mean daily MAP. \\ - Mean PRx. \\ - Mean daily PRx at CPPopt. \\ - Mean daily cerebral microdialysis lactate/pyruvate ratio. \\ - Brain tissue glucose as well as brain tissue oxygen tension (Pbt0 and $\mathrm{PbtO}_{2} / \mathrm{PaO}_{2}$ ratio). \\ Near infrared spectroscopy.}

CPP, cerebral perfusion pressure; CPPopt, optimal cerebral perfusion pressure; ICP, intracranial pressure; MAP, mean arterial blood pressure; PRx, pressure reactivity index; RAP, compensatory reserve index.

implemented in $\mathrm{ICM}+$ and investigated in a retrospective TBI data set by Liu et al. ${ }^{12}$ The algorithm has been further adapted to make it more suitable for prospective bed-side use.

At each time point, 36 PRx-CPP plots are generated from past data windows of increasing duration ranging from 2 to 8 hours, using incremental steps of $10 \mathrm{~min}$. Prior to that, CPP time series are preprocessed with a 5 min duration median filter and PRx data are Fisher transformed. ${ }^{16}$ Subsequently, all the data points are divided into groups corresponding to CPP bins of $5 \mathrm{~mm}$ $\mathrm{Hg}$ length, within $40-120 \mathrm{~mm} \mathrm{Hg}$ range of CPP values. For each bin, mean PRx and CPP values are used to fit a second order polynomial describing the theoretical U-shape, with its nadir determining CPPopt. This process is repeated for each progressively longer data window. Individual results undergo certain quality control criteria and the accepted values are combined using weighted average operation, as detailed below. The calculations are repeated every minute and the resulting time series is finally subjected to an exponentially weighted average (EWA) filter of 2 hours of duration (details below), forming the CPPopt time trend and giving the current CPPopt target recommendation.

The curve fitting process for each time window follows the algorithm described by Aries $e t a l^{10}$ with additional criteria as follows:

1. Each CPP bin must represent at least $3 \%$ of the total data count. In this way, CPP values that are very scarcely represented, likely due to short spikes or drops, but not to the physiological trend, will be disregarded.

2. At least $50 \%$ of the data in the time window must be included in the curve fit.

3. A PRx variation of at least 0.2 is mandated (thus rejecting flatter PRx-CPP curves).

4. The PRx range of interest is enforced to be between -0.3 and 0.6: the algorithm will not return any CPPopt value when PRx is always very high (indicating a complete loss of pressure reactivity) or always very low (pressure reactivity preserved at each CPP value).

5 . The coefficient of determination of the fitted curve $\mathrm{R}_{\text {full }}^{2}$ (calculated also for the bins excluded from the curve fitting process) must be at least 0.2.

The weights for combining the CPPopt calculations are given by the following formula:

$$
\text { weight }=\mathrm{R}_{\text {full }}^{2} * \begin{cases}1, & \text { shape }=\mathrm{P} \\ 0, & \text { shape }=\mathrm{NP}\end{cases}
$$

where $\mathrm{P}$ and NP stand for parabolic (U-shape curve) and non-parabolic (non-U shape curve), respectively. In this way, in the COGiTATE study only parabolic curves are taken into account.

The EWA weight is calculated as $(1-\alpha)^{k}$ where $k$ is the distance, in number of samples, from the current sample and $\alpha$ is set at 0.1 . In this way, more recent CPPopt values contribute more to the final calculation.

The curve fit and the weighting heuristics were chosen based on their performance in our retrospective data set, with regards to the resulting CPPopt time series lowest short term variability, ability to reject non-physiological values, and greatest discrimination from values generated from randomised, surrogate, signals (unpublished data).

The missing data limit of the calculation is set at $50 \%$, therefore at least 4 hours of continuously acquired data are necessary to generate the first CPPopt value.

\section{Assessment of compliance}

Compliance with the protocol will be assessed at 4 hourly CPP treatment reviews and by investigators from the research team three times per day. The 4-hour interval is a pragmatic choice and reflects results from visualisation studies which suggested that CPPopt changes over a time frame of 4-8hours, making the 4 hours an appropriate choice. ${ }^{17}$ To facilitate the compliance assessment, a custom module has been added to the ICM+ software that implements the alert and review system designed specifically for this trial. During the review time points, a CPP target is suggested by the software according to the protocol and depending on the arm. The treating clinicians may 
deviate from protocol but must provide their proposed target and clinical rationale for deviation in a structured short questionnaire presented by the software (see online supplementary files 1 and 2).

\section{Outcome measures \\ Feasibility}

Percentage of monitored time with measured CPP within a range of $5 \mathrm{~mm} \mathrm{Hg}$ above or below CPPopt.

The primary objective of the study is to demonstrate that it is clinically feasible to individualise CPP at CPPopt in TBI patients continuously over time, and demonstrate that we could achieve acceptable concordance with CPPopt. We also wish to explore whether a CPPoptguided strategy resulted in a significant difference in CPP compared with the controls. Specifically, we aim to assess whether the application of a CPPopt oriented protocol provides a greater percentage of time during which CPP is within $5 \mathrm{~mm} \mathrm{Hg}$ above or below the calculated CPPopt during the first 5 days of admission. Analysis of retrospective dataset in patients not managed according to the CPPopt concept showed that on average $30 \%$ of the monitored time CPP fell within $5 \mathrm{~mm} \mathrm{Hg}$ margin of CPPopt (unpublished data). This study was powered to target an increase of the monitored time by $20 \%$ (relative increase), meaning increase from $30 \%$ to $36 \%$ of the monitored time within the $5 \mathrm{~mm} \mathrm{Hg}$ margin of CPPopt, in the intervention group.

\section{Safety: daily TIL score}

The main secondary objective of the study is to demonstrate that targeting CPPopt is safe. Safety is defined in two domains. First, COGiTATE aims to assess whether a dynamic target is associated with excess haemodynamic support or organ failure. Second, the study will assess whether such a therapy leads to either worse or more difficult ICP control by driving cerebral oedema. This will be assessed by looking for an excess of (potentially burdensome) treatments to control ICP in the treatment arm as assessed by the use of the daily TIL score. ${ }^{18}$ A change in TIL score of $>3$ is representative of a significant escalation of TBI treatment from basic ICP management involving rescue therapies which are known to carry a clinically significant risk of harm. An increase of TIL score $>3$ is therefore expected to represent a clinically significant potentially harmful effect of CPPopt-guided treatment and the assessment of this will be the main secondary endpoint.

\section{Safety: safety and physiology variables}

Finally, we want to evaluate differences in physiological parameters between interventional and control arms (box 1). In particular, it is aimed to assess whether CPPoptguided therapy itself affects autoregulation indexes compared with the control group. Brain oxygenation and tissue chemistry will be assessed in those patients for which additional monitoring was considered clinically justifiable.

\section{Study population}

The study population consists of unconscious adult patients with severe TBI admitted to the ICU of three academic hospitals in whom invasive ICP monitoring is clinically indicated.

Inclusion criteria: (1) any adult severe TBI patient (age $>18$ years) requiring multimodality monitoring and ICP-directed therapy for at least 24 hours on the assessment of the recruiting team; (2) randomisation occurred within 24 hours after ICU admission; (3) informed consent/ agreement obtained by a legally authorised representative before inclusion, accordingly to the local legislations.

Exclusion criteria: (1) patients $<18$ years old; (2) known pregnancy; (3) moribund at presentation (eg, bilaterally absent pupillary responses); (4) patients with primary decompressive craniectomy (DC); (5) patients already enrolled in one other intervention study.

If patients are treated with a secondary DC, they will end the study as it is unknown how brain physiology changes after a DC and the calculation of CPPopt is poorly validated in such patients. Data up to the time of the DC will be retained for analysis. Once the study is terminated, the patients' CPP will be managed according to the BTF guidelines.

\section{Study arms and randomisation}

Patients are randomised to either standard CPP protocol therapy (control group) or to CPPopt-guided management of CPP (intervention group). Block randomisation by centre are used to ensure a uniform distribution of patients. Randomisation is done electronically on recruitment using a purpose built electronic Case Report Form (eCRF) hosted by the Clinical Trial Center Maastricht in Maastricht with a secure web interface.

Patient allocated to the control group are managed according to the most recent BTF guidelines ${ }^{2}$ which recommend maintaining CPP between 60 and $70 \mathrm{~mm}$ $\mathrm{Hg}$. In this group, online autoregulation information is hidden from the treating team although available to the study team for analysis.

Patients allocated to the intervention group are managed according so as to target CPPopt, when there is sufficient data for it to be available. If at the fixed review time points the CPPopt value is not available (eg, due to noisy or missing data) or it is outside of the safety limits (50-100 $\mathrm{mm} \mathrm{Hg}$ ), the protocol advises to follow a CPP target at the discretion of the clinician: this may be either the BTF guideline target or a previous target. As a safety precaution, the maximum difference in CPP target value suggested by the software at the new review time from the current target value has been limited to $\pm 10 \mathrm{~mm} \mathrm{Hg}$ to avoid potentially harmful jumps in CPP.

A flowchart of the patients' inclusion, randomisation and study procedures in the COGiTATE trial is presented in figure 1 .

\section{Sample size}

The sample size justification is based on the primary endpoint for the feasibility objective. The analyses will be 


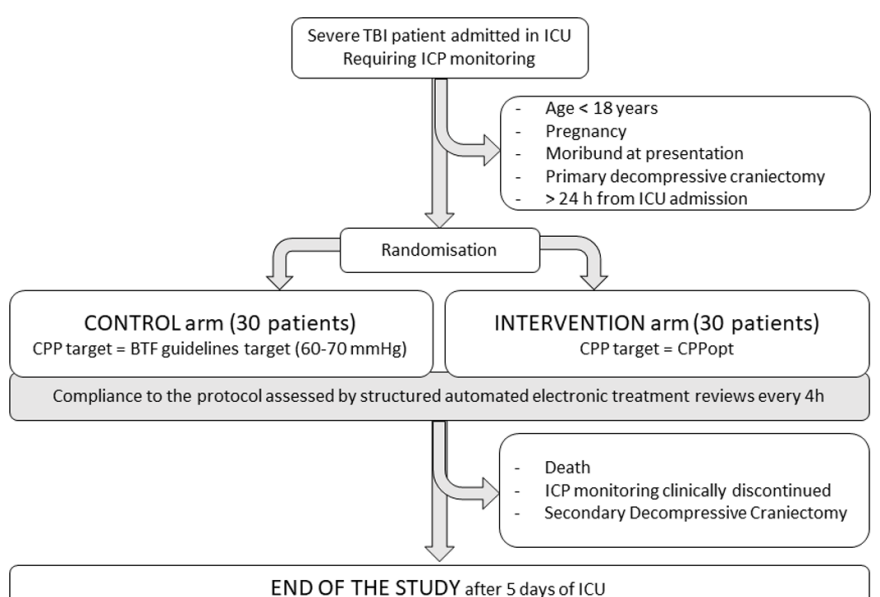

END OF THE STUDY after 5 days of ICU

Figure 1 Flowchart of the patients' inclusion, randomisation and study procedures in the COGiTATE trial. BTF, Brain Trauma Foundation; CPP, cerebral perfusionpressure; CPPopt, optimal cerebral perfusion pressure; ICP, intracranial pressure; ICU, intensive care unit; TBI, traumatic brain injury.

conducted according to the intention-to-treat principle, therefore all the enrolled and randomised patients will be included in the analysis, regardless of their compliance with the protocol. Sample sizes were determined using the $\mathrm{R}$ statistical language ${ }^{19}$ and the PWR package. ${ }^{20} \mathrm{~A}$ significance of $5 \%$ and power of $80 \%$ were assumed.

As described in the outcome measures paragraph, the primary endpoint for the main objective, feasibility, is the percentage of monitored time with measured CPP within a range of $5 \mathrm{~mm} \mathrm{Hg}$ above or below CPPopt. Retrospective analysis in unpublished data showed that on average patients spent a mean $(+\mathrm{SD})$ of $30 \%(+8 \%)$ of their monitored time with measured CPP within $5 \mathrm{~mm} \mathrm{Hg}$ of CPPopt. In the absence of prospective data, we powered COGiTATE study to target a relative increase in CPPopt target adherence by $20 \%$ (a pragmatic choice but justifiable on typical differences seen between good/poor outcome in the retrospective data), that is, from $30 \%$ to $36 \%$ of monitored time within the CPPopt target margin, while keeping the SD at $8 \%$. This produced a desired sample size of 56 , which was increased to 60 patients, to allow for dropout, technical problems or need for a non-parametric analysis.

The primary endpoint for the safety secondary objective is to assess whether there is an increase of the daily TIL score of $>3$ in the CPPopt treatment arm. We regard an increase of daily TIL of 3 as a significant effect as it represents a difference comparable to one treatment tier and in comparison with typical TIL variability from pilot data. Conversely, a TIL score increase of 2 can be expected in the intervention group because two points are counted for CPP-related therapy (vasopressor therapy/fluids), so this could be not interpreted as a significant worsening of TBI therapy. We estimate, for $80 \%$ power and $5 \%$ significance an averaged change in daily TIL score of 3 would require an $n=25$ in each arm (although this is a secondary endpoint and this estimation does not correct for multiple comparisons).

\section{Data storage}

High frequency ICP and ABP signals are recorded continuously and processed to obtain CPP and CPPopt. The values are subsequently transferred to secure local servers for offline analysis. Only the local study teams have access to the server.

Additional clinical and biochemical data (severity of injury and hospital course) use the patient's medical record as source data and are subsequently transcribed to the eCRFs with access limited to members of the study team only.

Imaging data and radiological reports obtained from the CT scans, are collected and stored on a secure local server.

The patients enrolled in the study and randomised, are assigned a study deidentification number that will be used in the data collection forms. An enrolment log is used in each centre to keep the link between the participants' identity and the study number. This log is secured in password-protected local servers to which only the local study coordinator and the members of the research team have access. The data transmitted to the data coordinating centre in Maastricht will be entirely deidentified.

Paper copies of the data, consent forms and protocols will be stored locally. Personal data will be destroyed after 1 year. Fifteen years after the completion of the study, all the paper study files and all the stored electronic files will be destroyed.

\section{Statistical analysis}

The data will be analysed on an intention-to-treat basis with all randomised patients included in the analysis. For the primary endpoint, the total time (in minutes) each patient had CPP values close $( \pm 5 \mathrm{~mm} \mathrm{Hg})$ to CPPopt will be calculated and analysed in a blinded manner by the study team. The fraction of time that CPP will be within $5 \mathrm{~mm} \mathrm{Hg}$ of CPPopt, will represent the primary analysis for this outcome. For secondary analysis, different deviation thresholds $(7.5$ and $10 \mathrm{~mm} \mathrm{Hg}$ ) will also be assessed. The two groups will be compared with an unpaired t-test (or a non-parametric equivalent in the event of a significant departure the normal distribution). Multivariable linear models will be used to control for the covariates centre, age, Glasgow Coma Score after resuscitation, pupil reactivity, presence of extracranial injury and primary craniotomy.

For the secondary safety endpoint, a t-test (or non-parametric equivalent) will be used for the statistical comparison of the TIL score, vasopressor and fluid use and organ dysfunction summary measures between the two groups. If necessary, multivariable linear regression analysis will be used to adjust for imbalances in main prognostic variables between the two groups.

For the other secondary safety and physiology endpoints, the results will be presented as mean (SD) for continuous 
variables and as frequencies (\%) for categorical variables. Intervention and control group will be compared using a t-test or non-parametric equivalent for continuous variables and $\chi^{2} /$ Fisher test for categorical variables.

\section{Patient and public involvement}

Improving outcomes from TBI is a known area of societal importance. ${ }^{3}$ Patients and public were not involved in the study design and, as patients in this study were unconscious, they could not be otherwise involved. The results will be disseminated through peer-reviewed publications and presentation at international conferences as well as by social media and the study website (www.cppopt.org) which also contains information for the public.

\section{ETHICS AND DISSEMINATION \\ Ethical considerations}

The trial is conducted according to the Declaration of Helsinki and the respective participating countries laws governing clinical research, and the principles of Good Clinical Practice.

Enrolled subjects, by definition, lack capacity to consent due to their level of consciousness at presentation. The informed consent procedure involves identifying a legally authorised representative of the patient, whose opinion is sought about the participant's wishes and feelings in relation to the project, and whether he/she would want to take part to the study. When available, a legally authorised representative (accordingly to the local laws) is then approached by a member of the clinical team, to inform them of the research study. If he/she agrees, a member of the research team will then provide further verbal and written explanation of the research study. If the family member/legal representative advises that the patient would want to take part in the study, the patient will be enrolled.

For this study, it was deemed physiologically important to begin CPPopt therapy as early as possible following the injury since pathobiological determinants of inflammation and oedema are likely to be determined in the first 24-48 hours after injury. Therefore, if a legally authorised representative is not identified within the first 12 hours after ICU admission, depending on the local country laws, the patient may be included via agreement with one of the treating clinicians not involved in the study (in UK), or using a deferred consent procedure (in Netherlands and Belgium). Following enrolment via deferred consent, if a legal representative does not agree for the patient to continue their participation in the study within 24 hours the patient will be withdrawn.

If the patient regains consciousness and capacity during their admission (ICU or hospital admission according to the local protocols), the patient will be approached to provide consent for their participation in the study. If the patient declines, they will be given the option to have data collected retained for analysis or destroyed completely.

\section{Safety considerations}

Safety of this study is independently monitored by an external clinical trials agency (Clinical Trial Center Maastricht, Oxfordlaan 70, $6229 \mathrm{EV}$ Maastricht (NL)) who will also have oversight of data quality. For this cohort of patients, a mortality rate of $25 \%$ and a severe disability rate of $30 \%$ is expected at baseline. However, it is unknown whether targeting CPPopt, which may typically be higher than a fixed range of $60-70 \mathrm{~mm} \mathrm{Hg}$ could drive further oedema or contusion expansion over the next days or lead to respiratory, renal or myocardial injury due to greater vasopressor requirements. Therefore, the followings are reported: increases in oedema or haemorrhages on repeated brain CT, troponin rise and/or ECG changes, NT-proBNP rise, significant serum creatinine rise and $\mathrm{PaO}_{2} / \mathrm{FiO}_{2}$ ratio $<300$. Severe adverse events and predefined adverse events that are commonly expected in TBI patients will also be recorded and reported. Glasgow Outcome Scale Extended at 6 months will also be collected as part of institutional practice or other observational studies.

\section{Dissemination}

The results of this study will be presented at international scientific conferences and in peer-reviewed journals, regardless of the trial outcome.

\section{Author affiliations}

${ }^{1}$ Clinical Neuroscience, University of Cambridge, Cambridge, UK

${ }^{2}$ Physiology and Transplantation, University of Milan, Milano, Italy

${ }^{3}$ Anaesthesia and Intensive Care,Policlinico San Martino, IRCCS for Oncology and

Neuroscience, University of Genoa, Genova, Italy

${ }^{4}$ Intensive Care, Maastricht Universitair Medisch Centrum+, Maastricht, The

Netherlands

${ }^{5}$ Division of Anaesthesia, University of Cambridge, Cambridge, UK

${ }^{6}$ Intensieve geneeskunde, Universitaire Ziekenhuizen Leuven, Leuven, Belgium

Acknowledgements The project is supported by an award from the European Society of Intensive Care Medicine awarded to MJA. DKM, AE, PS, MCz and MCa are supported by a European Union Framework Program 7 grant (CENTER-TBI; agreement no. 602150). DKM is supported by funding from the National Institute for Health Research (NIHR) UK through a Senior Investigator Award, the Cambridge Biomedical Research Centre, and the Global Health Research Group on Neurotrauma. GM is supported by the Research Foundation, Flanders (FWO) as Senior Clinical Investigator (1843118N). PJH is supported by the UK NIHR: Research Professorship, Cambridge Biomedical Research Centre and Global Health Research Group on Neurotrauma.The authors would like to thank E.Thellin, A.Kolias, A.Lalou, L. Gergele, I. Ng, A.Liberti, C. Turner, D. Bruyninckx, J.Lodewyck, I.Callebaut, F. Hermans, M. Borg and R Haeren for their support and useful discussions.

Contributors MJA, AE, PS, MC, JT, JD, MTC, CR, JGO, PH, DM, GM, BD and EB were involved in the study conception and developed the research design and methodology. EB, AE and PS drafted the manuscript. All authors edited drafts of the manuscript and approved the final manuscript.

Funding This study was supported by European Society of Intensive Care Medicine small investigator award (N/A: awarded to MJA).

Competing interests $\mathrm{PS}$ and $\mathrm{MCz}$ receive part of the licensing fees for multimodal brain monitoring software ICM+, licensed by Cambridge Enterprise Ltd, University of Cambridge, UK. There are no other conflicts of interest to declare.

Patient consent for publication Not required.

Ethics approval The research ethics committees of all the participating centres have approved the study protocols (Research Ethics Reference 17/LO/1119, Leuven B322201834820 and Maastricht NL60173.068.17). 
Provenance and peer review Not commissioned; externally peer reviewed.

Open access This is an open access article distributed in accordance with the Creative Commons Attribution Non Commercial (CC BY-NC 4.0) license, which permits others to distribute, remix, adapt, build upon this work non-commercially, and license their derivative works on different terms, provided the original work is properly cited, appropriate credit is given, any changes made indicated, and the use is non-commercial. See: http://creativecommons.org/licenses/by-nc/4.0/.

\section{REFERENCES}

1. Stocchetti N, Carbonara M, Citerio G, et al. Severe traumatic brain injury: targeted management in the intensive care unit. Lancet Neurol 2017;16:452-64.

2. Carney N, Totten AM, O'Reilly C, et al. Guidelines for the management of severe traumatic brain injury, fourth edition. Neurosurgery 2016;80:1

3. Maas AIR, Menon DK, Adelson PD, et al. Traumatic brain injury: integrated approaches to improve prevention, clinical care, and research. Lancet Neurol 2017;16:987-1048.

4. Menon DK, Ercole A. Critical care management of traumatic brain injury. In: Handbook of clinical neurology. Elsevier, 2017: 239-74.

5. Czosnyka M, Smielewski P, Kirkpatrick P, et al. Continuous assessment of the cerebral vasomotor reactivity in head injury. Neurosurgery 1997;41:11-19.

6. Donnelly J, Aries MJ, Czosnyka M. Further understanding of cerebral autoregulation at the bedside: possible implications for future therapy. Expert Rev Neurother 2015;15:169-85.

7. Czosnyka M, Brady K, Reinhard M, et al. Monitoring of cerebrovascular autoregulation: facts, myths, and missing links. Neurocrit Care 2009:10:373-86.

8. Zweifel C, Lavinio A, Steiner LA, et al. Continuous monitoring of cerebrovascular pressure reactivity in patients with head injury. Neurosurg Focus 2008;25:E2.

9. Steiner LA, Czosnyka M, Piechnik SK, et al. Continuous monitoring of cerebrovascular pressure reactivity allows determination of optimal cerebral perfusion pressure in patients with traumatic brain injury. Crit Care Med 2002;30:733-8.

10. Aries MJH, Czosnyka M, Budohoski KP, et al. Continuous determination of optimal cerebral perfusion pressure in traumatic brain injury*. Crit Care Med 2012;40:2456-63.

11. Needham E, McFadyen C, Newcombe V, et al. Cerebral perfusion pressure targets individualized to Pressure-Reactivity index in moderate to severe traumatic brain injury: a systematic review. $J$ Neurotrauma 2017;34:963-70.

12. Liu X, Maurits NM, Aries MJH, et al. Monitoring of optimal cerebral perfusion pressure in traumatic brain injured patients using a MultiWindow weighting algorithm. J Neurotrauma 2017;34:3081-8.

13. Dias C, Silva MJ, Pereira E, et al. Optimal cerebral perfusion pressure management at bedside: a single-center pilot study. Neurocrit Care 2015;23:92-102.

14. Jaeger M, Dengl M, Meixensberger J, et al. Effects of cerebrovascular pressure reactivity-guided optimization of cerebral perfusion pressure on brain tissue oxygenation after traumatic brain injury. Crit Care Med 2010;38:1343-7.

15. Depreitere B, Güiza F, Van den Berghe G, et al. Pressure autoregulation monitoring and cerebral perfusion pressure target recommendation in patients with severe traumatic brain injury based on minute-by-minute monitoring data. J Neurosurg 2014;120:1451-7.

16. Kelly S, Bishop SM, Ercole A. Statistical signal properties of the Pressure-Reactivity index (Prx). Acta Neurochir Suppl 2018;126:317-20.

17. Ercole A, Smielewski P, Aries MJH, et al. Visualisation of the 'Optima Cerebral Perfusion' Landscape in Severe Traumatic Brain Injury Patients. Acta Neurochir Supp/ 2018;126:55-8.

18. Zuercher P, Groen JL, Aries MJH, et al. Reliability and validity of the therapy intensity level scale: analysis of Clinimetric properties of a novel approach to assess management of intracranial pressure in traumatic brain injury. J Neurotrauma 2016;33:1768-74.

19. R Core Team. R: a language and environment for statistical computing. Vienna, Austria R Foundation for Statistical Computing; 2013. http://www.r-project.org/

20. Stephane Champely. pwr: basic functions for power analysis. R package version 1.2-1, 2017. Available: https://cran.r-project.org/ package $=$ pwr 